E. Sakai

Nagoya Math. J.

Vol. 38 (1970), 1-12

\title{
MEROMORPHIG OR HOLOMORPHIC COMPLETION OF A REINHARDT DOMAIN
}

\author{
EIICHI SAKAI \\ Dedicated to Professor Katuzi Ono on his 60th birthday
}

\section{Introduction}

In the theory of functions of several complex variables, the problem about the continuation of meromorphic functions has not been much investigated for a long time in spite of its importance except the deeper result of the continuity theorem due to E. E. Levi [4] and H. Kneser [3]. The difficulty of its investigation is based on the following reasons: we can not use the tools of not only Cauchy's integral formula but also the power series and there are indetermination points for the meromorphic function of many variables different from one variable. Therefore we shall also follow the Levi and Kneser's method and seek for the aspect of meromorphic completion of a Reinhardt domain in $\boldsymbol{C}^{n}$.

The main purpose of the present note is to prove that any meromorphic function in a Reinhardt domain $D$ in $\boldsymbol{C}^{n}$ is meromorphically continued to the envelope of holomorphy of $D$.

Kajiwara and the author [2] have proved this result for any domain over a Stein manifold. Therefore the above result is established naturally as the special case of [2]. However, for the elementary domain like the Reinhardt domain, it is desirable to give the direct and simple proof. For the case of $n=2$, Thullen [8] has discussed in detail such a completion. In the case of $n(\geqq 2)$ complex variables, we shall give a new proof about theorems on meromorphic completion by the only use of Continuity Theorem of Levi-Kneser (Kneser [3]).

If throughout this paper we replace the term "meromorphic (or meromorphy)" by "holomorphic (or holomorphy)", then the given theorems are

Received March 31, 1969 
all reduced to the well-known theorems on holomorphic completion obtained by Fabry, Faber, Hartogs and H. Cartan. So the new proof of their theorems is obtained by the only use of the continuity theorem of Hartogs.

\section{§1. Notations and definitions}

Let $\boldsymbol{C}^{n}(n \geqq 2)$ denote the $n$-dimensional complex number space which is the direct product of $n$ complex planes, each letter of $a, b, c, \cdots, w, z$ etc. denote a point of $\boldsymbol{C}^{n},\left(z_{1}, \cdots, z_{n}\right)$ denote coordinates of a point $z$, and $\left\{\left|z_{j}-a_{j}\right|<r_{j}\right\}_{j=1}^{n}$ denote a polydisc which is the direct product of $n$ discs $\left\{\left|z_{j}-a_{j}\right|<r_{j}\right\}, j=1, \cdots, n$.

A domain ${ }^{1)} D$ of $\boldsymbol{C}^{n}$ is called a Reinhardt domain with center at the point $c$ with coordinates $c_{1}, \cdots, c_{n}$ if it is invariant under the $n$-parameter transformation group $\left\{T\left(\theta_{1}, \cdots, \theta_{n}\right)\right\}$, each element of which is a biholomorphic mapping:

$$
T\left(\theta_{1}, \cdots, \theta_{n}\right): z_{j}^{\prime}=e^{i \theta_{j}}\left(z_{j}-c_{j}\right)+c_{j}, \quad j=1, \cdots, n,
$$

where $0 \leqq \theta_{j} \leqq 2 \pi, j=1, \cdots, n$.

The Reinhardt domain $D$ is called proper if the center $c$ is an interior point of $D$. If along with each point $z^{(0)} \in D$ there belong to the domain $D$ all the points $z$ for which

$$
\left|z_{j}-c_{j}\right| \leqq\left|z_{j}^{(0)}-c_{j}\right|, \quad j=1, \cdots, n,
$$

then the domain $D$ is called a complete Reinhardt domain. Of course, a complete Reinhardt domain is proper. If $D$ is a Reinhardt domain with center at the point $c$, then by means of a domain ${ }^{2)} \Delta$ in first closed quadrant $\left\{x_{j} \geqq 0\right\}_{j=1}^{n}$ of the $n$-dimensional real Euclid space $\boldsymbol{R}^{n}\left(x_{1}, \cdots, x_{n}\right), D$ is expressed by the form

$$
D=\left\{z \in C^{n} ;\left(\left|z_{1}-c_{1}\right|, \cdots,\left|z_{n}-c_{n}\right|\right) \in \Delta\right\} .
$$

We shall call such a domain $\Delta$ a domain of real expression of $D$. Let $D$ be a Reinhardt domain with center at the point $c$. If the domain $\Delta$ of real expression of $D$ is mapped onto the (geometrically) convex domain $\Delta^{*}$ of the Euclid space $\boldsymbol{R}^{n}\left(\xi_{1}, \cdots, \xi_{n}\right)$ by the transformation $\xi_{j}=\log \left|z_{j}-c_{j}\right|$,

1) A connected and open set $D$ of $\boldsymbol{C}^{n}$ is called a domain.

2) Here a domain $\Delta$ denotes a connected and relatively open set in first closed quadrant $\left\{x_{j} \geqq 0\right\}_{j=1}^{n}$. 
$j=1, \cdots, n$, then $D$ is said to have the logarithmically convex domain of real expression.

Finally, we shall show that the envelope of meromorphy of a proper Reinhardt domain $D$ is the least complete Reinhardt domain containing $D$ which has the logarithmically convex domain of real expression.

\section{§2. Lemmas}

First of all we shall introduce the continuity theorem obtained by Kneser [3] and Okuda-Sakai [5].

Continuity Theorem of Levi-Kneser. If $f(z)$ is meromorphic in a neighbourhood of the union of the sets $\left\{z_{1}=a_{1}, \cdots, z_{n-1}=a_{n-1},\left|z_{n}\right|=r\right\}^{3)}$ and $\left\{z_{1}=a_{1}^{(p)}, \cdots, z_{n-1}=a_{n-1}^{(p)},\left|z_{n}\right| \leqq r\right\}, p=1,2, \cdots$, then $f(z)$ can be meromorphically continued also to a neighbourhood of the set $\left\{z_{1}=a_{1}, \cdots\right.$, $\left.z_{n-1}=a_{n-1},\left|z_{n}\right| \leqq r\right\}$, where $\lim _{p \rightarrow \infty} a_{j}^{(p)}=a_{j}, j=1, \cdots, n-1$.

By a remark of Okuda-Sakai [5], this theorem is equivalent to the following

Continuation Theorem. If $f(z)$ is meromorphic in a neighbourhood of the union of the sets $\left\{\left|z_{1}-a_{1}\right| \leqq \rho, z_{2}=a_{2}, \cdots, z_{n-1}=a_{n-1},\left|z_{n}\right|=r\right\}$ and $\left\{z_{1}=a_{1}\right.$, $\left.z_{2}=a_{2}, \cdots, z_{n-1}=a_{n-1},\left|z_{n}\right| \leqq r\right\}$, then $f(z)$ can be meromorphically continued also to a neighbourhood of the set $\left\{\left|z_{1}-a_{1}\right| \leqq \rho, z_{2}=a_{2}, \cdots, z_{n-1}=a_{n-1},\left|z_{n}\right| \leqq r\right\}$.

Proof of equivalence. From the assumption there exists $\delta>0$ such that $f$ is meromorphic in a neighbourhood of $C_{\delta}=\left\{\left|z_{1}-a_{1}\right| \leqq \delta, z_{2}=a_{2}, \cdots\right.$, $\left.z_{n-1}=a_{n-1},\left|z_{n}\right| \leqq r\right\}$. Let $\delta_{0}$ denote the supremum of the set of positive numbers $\delta$ such that $f$ is meromorphic in a neighbourhood of $C_{\delta}$. Assume that $\delta_{0} \leqq \rho$. Let $b_{1}$ be an arbitrary point on the circle $\left\{\left|z_{1}-a_{1}\right|=\delta_{0}\right\}$ and $b_{1}^{(p)} \in\left\{\left|z_{1}-a_{1}\right|<\delta_{0}\right\} \quad(p=1,2, \cdots)$ be a sequence convergent to $b_{1}$ from the interior of the circle. Then $f$ is meromorphic in a neighbourhood of the union of the sets $\left\{z_{1}=b_{1}, z_{2}=a_{2}, \cdots, z_{n-1}=a_{n-1},\left|z_{n}\right|=r\right\}$ and $\left\{z_{1}=b_{1}^{(p)}\right.$, $\left.z_{2}=a_{2}, \cdots, z_{n-1}=a_{n-1},\left|z_{n}\right| \leqq r\right\}$. Hence $f$ can be meromorphically continued to a neighbourhood of $\left\{z_{1}=b_{1}, z_{2}=a_{2}, \cdots, z_{n-1}=a_{n-1}, \quad\left|z_{n}\right| \leqq r\right\}$ from Continuity Theorem of Levi-Kneser. By the arbitrariness of $b_{1}, f$ can be meromorphically continued to a neighbourhood of $C_{\delta_{0}}$. This contradicts the definition of $\delta_{0}$. Thus it must hold $\delta_{0}>\rho$ and the proof is completed.

3) The point set satisfying a property $P$ is denoted by $\{P\}$. 
Conversely, we shall show that Continuation Theorem implies Continuity Theorem of Levi-Kneser. From the assumption of Continuity Theorem there exists $\delta>0$ such that $f$ is meromorphic in a neighbourhood of the set $\left\{\left|z_{1}-a_{1}\right|<2 \delta, \cdots,\left|z_{n-1}-a_{n-1}\right|<2 \delta,\left|z_{n}\right|=r\right\}$. Let $p$ be a sufficiently large integer such that $a_{j}^{(p)} \in\left\{\left|z_{j}-a_{j}\right|<\delta\right\}, j=1, \cdots, n-1$. Then $f$ is meromorphic in a neighbourhood of the union of the sets $\left\{\left|z_{1}-a_{1}^{(p)}\right| \leqq \delta\right.$, $\left.z_{2}=a_{2}^{(p)}, \cdots, z_{n-1}=a_{n-1}^{(p)}, \quad\left|z_{n}\right|=r\right\}$ and $\left\{z_{1}=a_{1}^{(p)}, z_{2}=a_{2}^{(p)}, \cdots, z_{n-1}=a_{n-1}^{(p)}\right.$, $\left.\left|z_{n}\right|=r\right\}$. Consequently, from Continuation Theorem $f$ can be meromorphically continued to a neighbourhood of $\left\{\left|z_{1}-a_{1}^{(p)}\right| \leqq \delta, \quad z_{2}=a_{2}^{(p)}, \cdots\right.$, $\left.z_{n-1}=a_{n-1}^{(p)},\left|z_{n}\right| \leqq r\right\}$ and hence of $\left\{z_{1}=a_{1}, z_{2}=a_{2}^{(p)}, \cdots, z_{n-1}=a_{n-1}^{(p)},\left|z_{n}\right| \leqq r\right\}$. By the mathematical induction, we come to the conclusion.

We have given the proof of Continuation Theorem equivalent to Continuity Theorem of Levi-Kneser ([5]). Here we do not touch this proof.

Using Continuation Theorem, we obtain the following two lemmas.

Lemma 1. If $f(z)$ is meromorphic in the union ${ }^{4}$ of the domains $\left\{a_{1}<\left|z_{1}\right|<b_{1}\right.$, $\left.\left|z_{2}\right|<b_{2}, \cdots,\left|z_{n}\right|<b_{n}\right\}$ and $\left\{\left|z_{j}\right|<b_{j}^{\prime}\right\}_{j=1}^{n}$, then $f(z)$ can be meromorphically continued to the polydisc $\left\{\left|z_{j}\right|<b_{j}\right\}_{j=1}^{n}$, where $a_{1}, b_{j}$ and $b_{j}^{\prime}(j=1, \cdots, n)$ are real numbers satisfying the condition $0<a_{1}<b_{1}^{\prime} \leqq b_{1}$ and $0<b_{j}^{\prime} \leqq b_{j}(j=2, \cdots, n)$.

Proof. Take any point $z^{0}=\left(z_{1}^{0}, \cdots, z_{n}^{0}\right)$ in the polydisc $\left\{\left|z_{j}\right|<b_{j}^{\prime}\right\}_{j=1}^{n}$. Let $\varepsilon$ be any positive number satisfying the inequalities $a_{1}<b_{1}^{\prime}-\varepsilon$ and $b_{n}^{\prime}<b_{n}-\varepsilon$. From the assumption $f$ is meromorphic in a neighbourhood of union of the sets $\left\{\left|z_{1}\right|=b_{1}^{\prime}-\varepsilon, z_{2}=z_{2}^{0}, \cdots, z_{n-1}=z_{n-1}^{0},\left|z_{n}\right| \leqq b_{n}-\varepsilon\right\}$ and $\left\{\left|z_{1}\right| \leqq b_{1}^{\prime}-\varepsilon, z_{2}=z_{2}^{0}, \cdots, z_{n-1}=z_{n-1}^{0}, z_{n}=0\right\}$. Then $f$ can be meromorphically continued to the set $\left\{\left|z_{1}\right| \leqq b_{1}^{\prime}-\varepsilon, z_{2}=z_{2}^{0}, \cdots, z_{n-1}=z_{n-1}^{0}\right.$, $\left.\left|z_{n}\right| \leqq b_{n}-\varepsilon\right\}$ from Continuation Theorem. Since $z_{j}^{0}(j=2, \cdots, n-1)$ and $\varepsilon$ are chosen arbitrarily, $f$ can be also meromorphically continued to the polydisc $\left\{\left|z_{1}\right|<b_{1}^{\prime}, \cdots,\left|z_{n-1}\right|<b_{n-1}^{\prime},\left|z_{n}\right|<b_{n}\right\}$. By the use of mathematical induction, $f$ can be meromorphically continued to the polydisc $\left\{\left|z_{1}\right|<b_{1}^{\prime},\left|z_{2}\right|<b_{2}, \cdots,\left|z_{n}\right|<b_{n}\right\}$ at last. Observing that the union of the domains $\left\{a_{1}<\left|z_{1}\right|<b_{1},\left|z_{2}\right|<b_{2}, \cdots,\left|z_{n}\right|<b_{n}\right\}$ and $\left\{\left|z_{1}\right|<b_{1}^{\prime},\left|z_{2}\right|<b_{2}\right.$, $\left.\cdots,\left|z_{n}\right|<b_{n}\right\}$ is the polydisc $\left\{\left|z_{j}\right|<b_{j}\right\}_{j=1}^{n}$, we complete the proof of our Lemma.

4) Here the union of such domains is called the J-shaped Reinhardt domain. 
Lemma 2. If $f(z)$ is meromorphic in the union ${ }^{5)}$ of the domains $\left\{\left|z_{1}\right|<b_{1}\right.$, $\left.a_{2}<\left|z_{2}\right|<b_{2}, \cdots, a_{n}<\left|z_{n}\right|<b_{n}\right\}$ and $\left\{\left|z_{j}\right|<b_{j}^{\prime}\right\}_{j=1}^{n}$, then $f(z)$ can be meromorphically continued to the polydisc $\left\{\left|z_{j}\right|<b_{j}\right\}_{j=1}^{n}$, where $0<b_{1}^{\prime} \leqq b_{1}$ and $0<a_{j}<$ $b_{j}^{\prime} \leqq b_{j}(j=2, \cdots, n)$.

Proof. For the simplicity we shall prove this lemma in the case of $n=3$. Let $f$ be meromorphic in the union of domains $B=\left\{\left|z_{1}\right|<b_{1}, a_{2}\right.$ $\left.<\left|z_{2}\right|<b_{2}, a_{3}<\left|z_{3}\right|<b_{3}\right\}$ and $C=\left\{\left|z_{j}\right|<b_{j}^{\prime}\right\}_{j=1}^{3}$. If we take any point $z_{2}^{0}$ $\left(a_{2}<\left|z_{2}^{0}\right|<b_{2}^{\prime}\right)$ and any positive number $\varepsilon$ so that it holds $b_{1}^{\prime}<b_{1}-\varepsilon$ and $a_{3}<b_{3}^{\prime}-\varepsilon, f$ is meromorphic in a neighbourhood of the union of the sets $\left\{\left|z_{1}\right| \leqq b_{1}-\varepsilon, z_{2}=z_{2}^{0},\left|z_{3}\right|=b_{3}^{\prime}-\varepsilon\right\}$ and $\left\{z_{1}=0, z_{2}=z_{2}^{0},\left|z_{3}\right| \leqq b_{3}^{\prime}-\varepsilon\right\}$. Then considering the arbitrariness of $z_{2}^{0}$ and $\varepsilon$, we can find by Continuation Theorem that $f$ can be meromorphically continued to the domain $B_{1}=$ $\left\{\left|z_{1}\right|<b_{1}, a_{2}<\left|z_{2}\right|<b_{2}^{\prime},\left|z_{3}\right|<b_{3}^{\prime}\right\}$. Hence $f$ is meromorphic in $B \cup B_{1} \supset \widetilde{B}_{1}=$ $\left\{\left|z_{1}\right|<b_{1}, a_{2}<\left|z_{2}\right|<b_{2}^{\prime},\left|z_{3}\right|<b_{3}\right\}$. Further we can find by Lemma 1 that $f$ can be meromorphically continued to the polydisc $C_{2}=\left\{\left|z_{1}\right|<b_{1},\left|z_{2}\right|<b_{2}^{\prime}\right.$, $\left.\left|z_{3}\right|<b_{3}\right\}$ from the meromorphy of $f$ in $\widetilde{B}_{1} \cup C$. If the index 2 is replaced with the index $3, f$ can be also meromorphically continued to the polydisc $C_{3}=\left\{\left|z_{1}\right|<b_{1}, \quad\left|z_{2}\right|<b_{2}, \quad\left|z_{3}\right|<b_{3}^{\prime}\right\} . \quad$ Since $B \cup C_{2} \cup C_{3}=\left\{\left|z_{j}\right|<b_{j}\right\}_{j=1}^{3}$, we conclude that $f$ is meromorphic in the polydisc $\left\{\left|z_{j}\right|<b_{j}\right\}_{j=1}^{3}$.

Example 1. Consider the domain $D_{0}=\left\{\left|z_{j}\right|<1\right\}_{j=1}^{n}-\left\{\left(\left|z_{1}\right|-1\right)^{2}+\right.$ $\left.\sum_{j=2}^{n}\left(\left|z_{j}\right|-1 / 2\right)^{2} \leqq 1 / 16\right\}$. Such domain $D_{0}$ is a well-known example (BehnkeStein's example) of the domain in which the first Cousin problem is always solvable, but the second is not necessarily solvable. If $f$ is meromorphic in $D_{0}, f$ can be meromorphically continued to the unit polydisc $\left\{\left|z_{j}\right|<1\right\}_{j=1}^{n}$ from Lemma 2. Because $f$ is meromorphic in the union of the domains $\left\{\left|z_{1}\right|<1, \frac{3}{4}<\left|z_{2}\right|<1, \cdots, \frac{3}{4}<\left|z_{n}\right|<1\right\}$ and $\left\{\left|z_{1}\right|<\frac{3}{4},\left|z_{2}\right|<1, \cdots\right.$, $\left.\left|z_{n}\right|<1\right\}$.

Example 2. Consider the domain $D=\left\{\left|z_{n}\right|<\left|z_{n-1}\right|<\cdots<\left|z_{1}\right|<1\right\}$. This is a domain of holomorphy. But if $f$ is meromorphic in a neighbourhood of the closure $\bar{D}$ of $D$, there exists some $\delta(>0)$ so that $f$ can be meromorphically continued to the polydisc $\left\{\left|z_{j}\right|<1+\delta\right\}_{j=1}^{n}$.

5) Here the union of such domains is called the $\Gamma$-shaped Reinhardt domain. 
Now let us prove this in the case of $n=3$ for simplicity. If $f$ is meromorphic in the closure of $D=\left\{\left|z_{3}\right|<\left|z_{2}\right|<\left|z_{1}\right|<1\right\}$, there exists some $\delta(>0)$ so that $f$ is meromorphic in the union of the domains $B_{1}=\left\{\left|z_{1}\right|<1+\delta\right.$, $\left.\left|z_{2}\right|<\delta, \quad\left|z_{3}\right|<\delta\right\}, B_{2}=\left\{1-\delta<\left|z_{1}\right|<1+\delta,\left|z_{2}\right|<1+\delta, \quad\left|z_{3}\right|<\delta\right\}$ and $B_{3}=$ $\left\{1-\delta<\left|z_{1}\right|<1+\delta, 1-\delta<\left|z_{2}\right|<1+\delta,\left|z_{3}\right|<1+\delta\right\}$. Hence it is found by Lemma 1 that $f$ can be meromorphically continued to $\tilde{B}_{1}=\left\{\left|z_{1}\right|<\right.$ $\left.1+\delta,\left|z_{2}\right|<1+\delta,\left|z_{3}\right|<\delta\right\}$ from the meromorphy of $f$ in $B_{1} \cup B_{2}$ and then it is also found by Lemma 2 that $f$ can be meromorphically continued to $\left\{\left|z_{j}\right|<1+\delta\right\}_{j=1}^{3}$ from the meromorphy of $f$ in $\widetilde{B}_{1} \cup B_{3}$.

\section{§3. Removable singularities}

We note that the second theorem on removable singularities holds for meromorphic functions ([6]).

Proposition $^{6)}$. Let $V$ be an analytic set of complex dimension $n-2$, which is defined by the common zeros of two functions $g(z)$ and $h(z)$ holomorphic and locally coprime $e^{7)}$ in a domain $D$ of $\boldsymbol{C}^{n}$. If $f(z)$ is meromorphic in $D-V$, then $V$ is a set of removable singularities of $f(z)$.

This is an immediate result of Weierstrass preparation theorem and the following lemma obtained easily from Lemma 1.

Lemma 3. Let $F_{j}(j=1,2)$ denote a closed set on the $z_{j}$-plane, where $F_{1}$ lies entirely in $\left\{\left|z_{1}\right|<r_{1}\right\}$ and $\left\{\left|z_{2}\right|<r_{2}\right\}-F_{2}$ is not empty. If $f(z)$ is meromorphic in the set of all those points in a polydisc $\left\{\left|z_{j}\right|<r_{j}\right\}_{j=1}^{n}$, for which $z_{1} \notin F_{1}$ or $z_{2} \notin F_{2}$, then $f(z)$ can be meromorphically continued to the complete polydisc $\left\{\left|z_{j}\right|<r_{j}\right\}_{j=1}^{n}$.

Proof. Denote by $D_{j}$ the disc $\left\{\left|z_{j}\right|<r_{j}\right\}$ on the $z_{j}$-plane, $(j=1, \cdots, n)$. From the assumption $f$ is meromorphic in $\left[\left(D_{1}-F_{1}\right) \times D_{2} \times \cdots \times D_{n}\right] \cup$ $\left[D_{1} \times\left(D_{2}-F_{2}\right) \times D_{3} \times \cdots \times D_{n}\right]$. Since the meromorphy is invariant under the biholomorphic mapping, it may be assumed that $D_{2}-F_{2}$ contains the origin in the $z_{2}$-plane. Then there exist some positive numbers $\rho\left(<r_{1}\right)$ and $\delta\left(<r_{2}\right)$ so that $f$ is meromorphic in the domain $\left\{\rho<\left|z_{1}\right|<r_{1},\left|z_{2}\right|<r_{2}\right.$, $\left.\cdots,\left|z_{n}\right|<r_{n}\right\} \cup\left\{\left|z_{1}\right|<r_{1},\left|z_{2}\right|<\delta,\left|z_{3}\right|<r_{3}, \cdots,\left|z_{n}\right|<r_{n}\right\}$. Therefore

6) Results of this type are stated in Behnke-Thullen [1] without proofs; for example, see "Folgerung 1", p. 50.

7) Functions $g(z)$ and $h(z)$ not all of which are identically zero, are called locally coprime in $D$ when $g$ and $h$ have no common divisors other than the units (i.e. factors of 1 ) in a neighbourhood of each point of $D$. 
it is concluded from Lemma 1 that $f$ can be meromorphically continued to the polydisc $\left\{\left|z_{j}\right|<r_{j}\right\}_{j=1}^{n}$.

\section{§4.' Radius of Meromorphy}

In [5] we proved the equivalence of Continuation Theorem and the fact that the logarithm of the radius of meromorphy is superharmonic ${ }^{8}$. If we could obtain another proof of the latter fact, then the above equivalence would give a new proof of Continuity Theorem of Levi-Kneser.

Let $f\left(w_{1}, \cdots, w_{n}, z\right)$ be meromorphic in a neighbourhood of the set $\left\{w=\left(w_{1}, \cdots, w_{n}\right) \in D, z=c\right\}$, where $D$ is a domain in the space $C^{n}(w)$. For each point $w^{0}$ in $D$, let $R\left(w^{0}\right)$ denote the supremum of the set of radii $r$ such that $f$ is meromorphic in $(w, z)$ in a neighbourhood of the set $\left\{w=w^{0},|z-c|<r\right\}$. Then $R\left(w^{0}\right)$ is called the radius of meromorphy of $f$ at a point $w^{0}$ with center at the point $c$.

THEOREM 1. If $R\left(w_{1}, \cdots, w_{n}\right)$ is the radius of meromorphy of a meromorphic function $f\left(w_{1}, \cdots, w_{n}, z\right)$ at a point $w$, then for a fixed point $\left(w_{2}^{0}, \cdots, w_{n}^{0}\right)$ $\log R\left(w_{1}, w_{2}^{0}, \cdots, w_{n}^{0}\right)$ is superharmonic in $w_{1}$. Further $\log R\left(w_{1}, \cdots, w_{n}\right)$ is plurisuperharmonic in $\left(w_{1}, \cdots, w_{n}\right)$.

A proof of the first statement of this theorem was given in [5]. To prove the second statement, it is enough to show that if the definition domain $D$ of $R(w)$ is cut by any analytic plane $E$ :

$w_{j}=a_{j} t+b_{j}\left(a_{j}, b_{j}\right.$ constant, $t$ complex parameter $), j=1, \cdots, n$, then $\log R\left(a_{1} t+b_{1}, \cdots, a_{n} t+b_{n}\right)$ is always superharmonic in $t$ on each connected component of $D \cap E$. We can reduce easily to the first half by a unitary transformation of $w_{1}$-axis onto $E$, considering that the meromorphy is invariant under a biholomorphic mapping.

In fact, let a unitary transformation of $w_{1}$-axis onto $E$ be

$$
w=\zeta U+b,
$$

where $w=\left(w_{1}, \cdots, w_{n}\right), \zeta=\left(\zeta_{1}, \cdots, \zeta_{n}\right)$ and $b=\left(b_{1}, \cdots, b_{n}\right)$ are the $1 \times n$ matrices, and $U$ is an $n \times n$ unitary matrix. Let $D^{*}$ be the image of $D$ by the transformation (1). Let $f^{*}(\zeta, z)$ and $R^{*}(\zeta)$ denote the transformed function of $f(w, z)$ by (1) and the radius of meromorphy of $f^{*}$ at each point

8) This fact is stated without proof and used essentially in W. Rothstein: Ein neuer Beweis des Hartogsschen Hauptsatzes und seine Ausdehnung auf meromorphe Funktionen, Math. Z., 53 (1950), 84-95. 
$\zeta \in D^{*}$ with center at the point $c$, respectively. Then it holds $R(\zeta U+b)=$ $R^{*}(\zeta)$ in $D^{*}$. Now, from the first statement of Theorem $1 \log R^{*}\left(\zeta_{1}, 0, \cdots, 0\right)$ is superharmonic in $\zeta_{1}$. Therefore $\log R\left(a_{1} t+b_{1}, \cdots, a_{n} t+b_{n}\right)$ is superharmonic in $t$, when $\log R^{*}\left(\zeta_{1}, 0, \cdots, 0\right)$ is returned to $w$-space by the transformation (1).

\section{§5. Reinhardt domains}

In what follows, we shall treat proper Reinhardt domains with center at the origin.

First of all we shall discuss the meromorphic continuation concerning meromorphic functions in such a domain of $\boldsymbol{C}^{n}$. In the case of $n=2$, Thullen [8] has investigated in detail such a continuation. In the general case of $n$ complex variables, we shall give another proof about theorems on meromorphic completion by the only use of Continuation Theorem.

By the use of Lemma 2, we obtain easily the following theorem which corresponds to the expansion theorem of $\mathrm{H}$. Cartan in the case of holomorphic functions.

THEOREM 2. If $f(z)$ is meromorphic in a proper Reinhardt domain D, then $f(z)$ can be meromorphically continued to the least complete Reinhardt domain containing $D$.

Proof. Now let $z^{0}$ be any point of $D$. Without loss of generality we may assume that each coordinate of $z^{0}$ is nonzero. Since $D$ contains the origin and is connected, we can join a neighbourhood $\left\{\left|z_{j}\right|<b_{j}^{0}\right\}_{j=1}^{n}(\subset D)$ of the origin with $z^{0}$ by a finite number of ring polydomains $Q_{k}=\left\{a_{j}^{(k)}<\right.$ $\left.\left|z_{j}\right|<b_{j}^{(k)}\right\}_{j=1}^{n} \subset D, \quad(k=1,2, \cdots, m)$, where $\left\{\left|z_{j}\right|<b_{j}^{0}\right\}_{j=1}^{n} \cap Q_{1} \neq \phi, Q_{k} \cap Q_{k+1} \neq \phi$ $(k=1,2, \cdots, m-1)$ and $z^{0} \in Q_{m}$.

We must show that $f$ can be meromorphically continued to a neighbourhood of $\left\{\left|z_{j}\right| \leqq\left|z_{j}^{0}\right|\right\}_{j=1}^{n}$. For this purpose it is enough to prove that $f$ can be meromorphically continued to the polydisc $\left\{\left|z_{j}\right|<b_{j}\right\}_{j=1}^{n}$, provided that $f$ is meromorphic in $\left\{\left|z_{j}\right|<b_{j}^{0}\right\}_{j=1}^{n} \cup\left\{a_{j}<\left|z_{j}\right|<b_{j}\right\}_{j=1}^{n}, \quad\left(a_{j}<b_{j}^{0} \leqq b_{j}, j=\right.$ $1,2, \cdots, n)$.

For the simplicity, we shall show this in the case of $n=3$. At first it is easily seen that the union of domains $\left\{\left|z_{j}\right|<b_{j}^{0}\right\}_{j=1}^{3}$ and $B=\left\{a_{j}<\right.$ $\left.\left|z_{j}\right|<b_{j}\right\}_{j=1}^{3}$ contains the domain $\left\{\left|z_{1}\right|<b_{1}, \quad a_{2}<\left|z_{2}\right|<b_{2}^{0}, \quad a_{3}<\left|z_{3}\right|<b_{3}^{0}\right\}$. Hence from Lemma 2, $f$ can be meromorphically continued to the polydisc $\left\{\left|z_{1}\right|<b_{1},\left|z_{2}\right|<b_{2}^{0}, \quad\left|z_{3}\right|<b_{3}^{0}\right\}$. Further since the union of this polydisc 
and $B$ contains the domain $\left\{a_{1}<\left|z_{1}\right|<b_{1}, \quad\left|z_{2}\right|<b_{2}, a_{3}<\left|z_{3}\right|<b_{3}^{0}\right\}, f$ can be meromorphically continued to the polydisc $C_{3}=\left\{\left|z_{1}\right|<b_{1},\left|z_{2}\right|<b_{2}\right.$, $\left.\left|z_{3}\right|<b_{3}^{0}\right\}$ by the use of Lemma 2 again. In the similar manner, $f$ can be meromorphically continued to the polydisc $C_{2}=\left\{\left|z_{1}\right|<b_{1},\left|z_{2}\right|<b_{2}^{0},\left|z_{3}\right|<b_{3}\right\}$ and $C_{1}=\left\{\left|z_{1}\right|<b_{1}^{0},\left|z_{2}\right|<b_{2}, \quad\left|z_{3}\right|<b_{3}\right\}$. Since $B \cup C_{1} \cup C_{2} \cup C_{3}=\left\{\left|z_{j}\right|<b_{j}\right\}_{j=1}^{3}$, we complete the proof of this theorem.

Theorem 3. If $f(z)$ is meromorphic in a proper Reinhardt domain D, then $f(z)$ can be meromorphically continued to the least complete Reinhardt domain containing $D$ which has the logarithmically convex domain of real expression.

Proof. By Theorem 2, it may be assumed that $D$ is a complete Reinhardt domain. Let $\Delta$ and $\Delta^{*}$ denote the domain of real expression of $D$ and the image of $\Delta$ contained in $\boldsymbol{R}^{n}(\xi)$ by the transformation

$$
\xi_{j}=\log \left|z_{j}\right|, \quad j=1,2, \cdots, n,
$$

respectively. Take any two points $\alpha=\left(\alpha_{1}, \cdots, \alpha_{n}\right), \beta=\left(\beta_{1}, \cdots, \beta_{n}\right)$ in $\Delta^{*}$. If $\xi=\left(\xi_{1}, \cdots, \xi_{n}\right)$ is any point on the line segment, which joins $\alpha$ and $\beta$, it is represented by the form

$$
\xi=\theta \alpha+(1-\theta) \beta, \quad(0 \leqq \theta \leqq 1),
$$

where $\theta \alpha$ denotes the scalar multiplication $\left(\theta \alpha_{1}, \cdots, \theta \alpha_{n}\right)$. Eliminating $\theta$ in (3), we have the following representation by the components of $\xi$ :

$$
\frac{\xi_{1}-\beta_{1}}{\alpha_{1}-\beta_{1}}=\cdots=\frac{\xi_{n-1}-\beta_{n-1}}{\alpha_{n-1}-\beta_{n-1}}=\frac{\xi_{n}-\beta_{n}}{\alpha_{n}-\beta_{n}} \text {. }
$$

Here we note that $\xi_{k}=\beta_{k}$ if $\alpha_{k}=\beta_{k}$ for some $k(1 \leqq k \leqq n)$.

Let $z=\left(z_{1}, \cdots, z_{n}\right), a=\left(a_{1}, \cdots, a_{n}\right)$ and $b=\left(b_{1}, \cdots, b_{n}\right)$ denote one of the inverse images of $\xi, \alpha$ and $\beta$ by the transformation (2), respectively. Then it is sufficient to prove that any meromorphic function $f$ in $D$ can be meromorphically continued to a domain which contains a point $z$ corresponding to $\xi$ in (3). Since $D$ is a complete Reinhardt domain, this is clear in the case $\alpha_{j} \leqq \beta_{j}(j=1, \cdots, n)$ or $\alpha_{j} \geqq \beta_{j}(j=1, \cdots, n)$ and hence it will be sufficient to prove this in the case where there exist two integers $j, k(1 \leqq j, k \leqq n, j \neq k)$ with $\alpha_{j}<\beta_{j}$ and $\alpha_{k}>\beta_{k}$. Then without loss of generality we may assume

$$
\alpha_{1}<\beta_{1} \text { and } \alpha_{n}>\beta_{n}
$$


Denote by $\left(\xi_{1}, \cdots, \xi_{n-1}, \beta_{n}\right)$ any point on the line segment

$$
\frac{\xi_{1}-\beta_{1}}{\alpha_{1}-\beta_{1}}=\cdots=\frac{\xi_{n-1}-\beta_{n-1}}{\alpha_{n-1}-\beta_{n-1}}, \quad \xi_{n}=\beta_{n},
$$

which joins two points $\left(\alpha_{1}, \cdots, \alpha_{n-1}, \beta_{n}\right)$ and $\left(\beta_{1}, \cdots, \beta_{n-1}, \beta_{n}\right)$, and further denote by $R\left(z_{1}, \cdots, z_{n-1}\right)$ the radius of meromorphy of $f$ at a point $\left(z_{1}, \cdots, z_{n-1}\right)$ with center at the origin $z_{n}=0$, where $f$ is a meromorphic function in $D$ and $\left(z_{1}, \cdots, z_{n-1}\right)$ is a point corresponding to the point $\left(\xi_{1}, \cdots, \xi_{n-1}\right)$. Then it will be sufficient to prove the inequality

$$
R\left(z_{1}, \cdots, z_{n-1}\right)>\left|z_{n}\right|
$$

for a point $z=\left(z_{1}, \cdots, z_{n-1}, z_{n}\right)$ corresponding to any point $\xi$ on the line segment $\left(3^{\prime}\right)$.

Since $D$ is a complete Reinhardt domain, it holds for any real $\theta_{j}(0 \leqq$ $\left.\theta_{j} \leqq 2 \pi, j=1, \cdots, n-1\right)$

$$
R\left(z_{1}, \cdots, z_{n-1}\right)=R\left(z_{1} e^{i \theta_{1}}, \cdots, z_{n-1} e^{i \theta_{n-1}}\right),
$$

and the following inequalities are obtained:

$$
R\left(a_{1}, \cdots, a_{n-1}\right)>\left|a_{n}\right| \text { and } R\left(b_{1}, \cdots, b_{n-1}\right)>\left|b_{n}\right| .
$$

We divide the proof of (6) into two cases $n=2$ and $n>2$.

(a) Case of $n=2$. Since $\log R\left(z_{1}\right)$ is superharmonic in $\left\{\left|z_{1}\right| \leqq\left|b_{1}\right|\right\}$ from Theorem 1 and $\xi_{1}=\log \left|z_{1}\right|$ is harmonic in $\left\{\left|a_{1}\right| \leqq\left|z_{1}\right| \leqq\left|b_{1}\right|\right\}$, then the function

$$
u\left(z_{1}\right)=\log R\left(z_{1}\right)-\beta_{2}-\frac{\alpha_{2}-\beta_{2}}{\alpha_{1}-\beta_{1}}\left(\xi_{1}-\beta_{1}\right)
$$

is superharmonic in $\left\{\left|a_{1}\right| \leqq\left|z_{1}\right| \leqq\left|b_{1}\right|\right\}$, where $\alpha_{j}=\log \left|a_{j}\right|, \quad \beta_{j}=\log \left|b_{j}\right|$ $(j=1,2)$ and $\xi_{1}=\log \left|z_{1}\right|$. By the inequalities (7), $u\left(z_{1}\right)$ takes the positive value on $\left\{\left|z_{1}\right|=\left|a_{1}\right|\right\}$ and $\left\{\left|z_{1}\right|=\left|b_{1}\right|\right\}$. Therefore by the minimum principle it holds $u\left(z_{1}\right)>0$ in $\left\{\left|a_{1}\right|<\left|z_{1}\right|<\left|b_{1}\right|\right\}$ and it implies

$$
\log R\left(z_{1}\right)>\beta_{2}+\frac{\alpha_{2}-\beta_{2}}{\alpha_{1}-\beta_{1}}\left(\xi_{1}-\beta_{1}\right)
$$

Since the right hand side of the above inequality is equal to $\xi_{2}$ and hence to $\log \left|z_{2}\right|$ by $\left(3^{\prime}\right)$, the inequality (6) establishes.

(b) Case of $n>2$. For the simplicity we assume that $\alpha_{j} \leqq \beta_{j}(j=2$, $\cdots, n-1)$ with (4). From Theorem $1 \log R\left(z_{1}, \cdots, z_{n-1}\right)$ is plurisuperharmonic in $\left\{\left|z_{j}\right| \leqq\left|b_{j}\right|\right\}_{j=1}^{n-1}$. A mapping $\varphi$ : 
(8)

$$
\left\{\begin{array}{l}
z_{1}=t \\
z_{j}=\exp \left\{\beta_{j}+\frac{\alpha_{j}-\beta_{j}}{\alpha_{1}-\beta_{1}}\left(\log t-\beta_{1}\right)\right\}, \quad j=2, \cdots, n-1,
\end{array}\right.
$$

give the holomorphic mapping from $\left\{\left|a_{1}\right| \leqq|t| \leqq\left|b_{1}\right|\right\}$ onto $\left\{\left|a_{j}\right| \leqq\left|z_{j}\right| \leqq\right.$ $\left.\left|b_{j}\right|\right\}_{j=1}^{n-1}$. If the radius $R\left(z_{1}, \cdots, z_{n-1}\right)$ of meromorphy is transformed by $\varphi$, then $f(t)=\log R\left(z_{1}, \cdots, z_{n-1}\right)$ is a superharmonic function of $t$ in $\left\{\left|a_{1}\right| \leqq|t| \leqq\left|b_{1}\right|\right\}$.

Let us consider the behavior of the function

$$
\log R\left(z_{1}, \cdots, z_{n-1}\right)-\beta_{n}-\frac{\alpha_{n}-\beta_{n}}{\alpha_{1}-\beta_{1}}\left(\log \left|z_{1}\right|-\beta_{1}\right)
$$

on the line segment (5). Denoting by $u(t)$ the transformed function of (9) by (8) for the purpose, we may consider it in $\left\{\left|a_{1}\right| \leqq|t| \leqq\left|b_{1}\right|\right\}$. Then it can be seen easily that $u(t)$ is superharmonic in $\left\{\left|a_{1}\right| \leqq|t| \leqq\left|b_{1}\right|\right\}$. Since $u\left(a_{1}\right)=\log R\left(a_{1}, \cdots, a_{n-1}\right)-\beta_{n}-\frac{\alpha_{n}-\beta_{n}}{\alpha_{1}-\beta_{1}}\left(\alpha_{1}-\beta_{1}\right)=\log R\left(a_{1}, \cdots, a_{n-1}\right)-\alpha_{n}>0$ and $u\left(b_{1}\right)=\log R\left(b_{1}, \cdots, b_{n-1}\right)-\beta_{n}>0$ from (7), it holds by the minimum principle $u(t)>0$ in $\left\{\left|a_{1}\right|<|t|<\left|b_{1}\right|\right\}$, and this implies

$$
f(t)=\log R\left(z_{1}, \cdots, z_{n-1}\right)>\beta_{n}+\frac{\alpha_{n}-\beta_{n}}{\alpha_{1}-\beta_{1}}\left(\xi_{1}-\beta_{1}\right) .
$$

From $\left(3^{\prime}\right)$ the right hand side of the above inequality is equal to $\xi_{n}$ and hence to $\log \left|z_{n}\right|$. Thus the inequality (6) is proved.

Now, a complete Reinhardt domain $\tilde{D}$ which has the logarithmically convex domain of real expression, is a domain of holomorphy. For we can construct easily a power series $f(z)$ absolutely convergent in $\tilde{D}$, so that $\tilde{D}$ is the domain of existence of $f(z)$. Consequently, it is concluded that the envelope of meromorphy of a proper Reinhardt domain $D$ coincides with the envelope of holomorphy of $D$.

\section{REFERENCES}

[1] Behnke, H., und P. Thullen, Theorie der Funktionen mehrerer komplexer Veränderlichen, Erg. d. Math. u. i. Grenzgeb. III, 3 (1934).

[2] Kajiwara, J., and E. Sakai, Generalization of Levi-Oka's theorem concerning meromorphic functions, Nagoya Math. J., 29 (1967), 75-84.

[ 3 ] Kneser, H., Ein Satz über die Meromorphiebereiche analytischer Funktionen von mehreren Veränderlichen, Math. Ann., 106 (1932), 648-655.

[4] Levi, E. E., Studii sui punti singolari essenziali delle funzioni analitiche de due o più variabili complesse, Ann. Mat. Pura Appl. (3), 17 (1910), 61-87. 
[5] Okuda, H., and E. Sakai, On the continuation theorem of Levi and the radius of meromorphy, Mem. Fac. Sci. Kyushu Univ. (A), 11 (1957), 65-73.

[6] Sakai, E., A note on meromorphic functions in several complex variables, Mem. Fac. Sci. Kyushu Univ. (A), 11 (1957), 75-80.

[ 7 ] Sakai, E., Problem of quotient representation and meromorphic completion, Sci. Rep. Kanazawa Univ., 11 (1966), 93-97.

[8] Thullen, P., De la theoria de las functiones analíticas de varias variables complejas. Dominios de regularidad y dominios meromorfia de Reinhardt, Revista Unión Mat. Argentina, 11 (1945), 33-46.

Department of Mathematics, Kanazawa University 